\title{
A kind of unified super efficiency via Assumption (A) and applications in vector optimization
}

\author{
Hui Guo ${ }^{1,2^{*}}$ and Ke Quan Zhao ${ }^{2}$
}

\section{"Correspondence:}

guoguofly@163.com

${ }^{1}$ College of Sciences, Shanghai

University, Shanghai, 200444, China

${ }^{2}$ School of Mathematical Sciences,

Chongqing Normal University,

Chongqing, 401331, China

\section{Springer}

\begin{abstract}
In this paper, we propose a new kind of S-super efficiency of vector optimization problems with set-valued maps via Assumption (A), which unifies exact and approximate super efficiency well known in the literature. Furthermore, we also introduce the concept of nearly S-subconvexlikeness via Assumption (A) and discuss some relations with other types of generalized convexity. Moreover, we establish some scalarization theorems and Lagrange multiplier theorems of S-super efficiency under the nearly S-subconvexlikeness.
\end{abstract}

MSC: $90 \mathrm{C} 26 ; 90 C 29 ; 90 C 30$

Keywords: vector optimization with set-valued maps; S-super efficiency; nearly S-subconvexlikeness; scalarization; Lagrange multipliers

\section{Introduction}

Borwein and Zhuang introduced the concept of super efficient solutions of vector optimization problems in normed linear spaces and established a scalarization theorem and Lagrange multiplier theorem for super efficiency of convex vector optimization in $[1,2]$. Then Zheng generalized the concept of super efficient points from normed spaces to real locally convex topological vector spaces in [3]. With the development of set-valued analysis, some researchers studied the super efficient solutions for vector optimization problems with set-valued maps. Especially, Rong and Wu [4] and Mehra [5] investigated super efficient solutions of vector optimization problems with set-valued maps in normed spaces under the cone-convexlikeness and the nearly cone-convexlikeness of set-valued maps, respectively. Qiu and Fu [6] studied the connectedness of super efficient solution sets of vector optimization problems with set-valued maps under the cone-convexlikeness. Xia and Qiu [7] studied the super efficiency under the nearly cone-subconvexlikeness of set-valued maps.

On the other hand, the concepts of approximate solutions play an important role when a vector optimization problem has no exact solutions. Kutateladze [8] introduced the notion of an approximate solution firstly. Then many researchers extended the notions of approximate solutions of vector-valued optimization problems to set-valued optimization problems. Rong and $\mathrm{Wu}[9]$ introduced $\varepsilon$-weakly minimal solutions of vector optimization problems. Ling $[10,11]$ introduced $\varepsilon$-super efficient solutions of vector optimization

(c) 2015 Guo and Zhao. This article is distributed under the terms of the Creative Commons Attribution 4.0 International License (http://creativecommons.org/licenses/by/4.0/), which permits unrestricted use, distribution, and reproduction in any medium, provided you give appropriate credit to the original author(s) and the source, provide a link to the Creative Commons license, and indicate if changes were made. 
problems and established the scalarization theorem, the Lagrange multiplier theorem, the $\varepsilon$-saddle points theorem, and the duality theorem for $\varepsilon$-super efficient solution under the assumption of the nearly generalized cone-subconvextikeness for set-valued maps. Tuan [12] studied $\varepsilon$-Benson efficient solutions of vector optimization problems in locally convex topological spaces.

Recently, Chicco et al. [13] introduced the concepts of improvement sets and $E$-optimal points and investigated the existence of this solution in $\mathbb{R}^{n}$. Furthermore, Gutiérrez et al. extended the notion of improvement sets and $E$-optimal points to a general (nonnecessarily Pareto) quasi-ordered linear space and obtained some necessary and sufficient conditions for $E$-optimal solutions of vector optimization problems through scalarization in [14]. Zhao et al. [15-17] investigated $E$-optimal solutions and $E$-Benson properly efficient solutions of set-valued optimization problems based on improvement sets in real locally convex Hausdorff topological vector spaces. Gutiérrez et al. proposed $(C, \varepsilon)$-efficient solutions via co-radiant sets, which extended and unified some known different notions of approximate solution in [18], and they obtained some characterizations of this kind of approximate solution by linear scalarization in [19]. Under the assumption of generalized subconvexlikeness and by using co-radiant sets, Gao et al. presented an alternative theorem of set-valued maps, and then derived scalarization theorems and Lagrange multiplier theorems for approximate solutions of vector optimization problems with set-valued maps in [20]. Moreover, Flores-Bazán and Hernández proposed Assumption (A) and Assumption (B) and obtained a complete scalarization in [21].

Motivated by the work of [15, 21-23], in this paper, we introduce a kind of unified super efficiency of vector optimization problems with set-valued maps, which is named $S$-super efficiency via Assumption (A) in real locally convex Hausdorff topological vector spaces. We also present the concept of nearly $S$-subconvexlikeness of set-valued maps. As applications, we establish some scalarization theorems and Lagrange multiplier theorems of $S$-super efficiency for vector optimization problems with set-valued maps.

\section{Preliminaries}

In this paper, let $X$ be a linear space, $Y$ and $Z$ be two real locally convex Hausdorff topological vector spaces with topological dual spaces $Y^{*}$ and $Z^{*}$, respectively. For a subset $M$ of $Y$, we denote the topological interior, topological closure, complement and topological boundary of $M$ by int $M, \operatorname{cl} M, \mathcal{C}(M)$, and $\partial M$. For any $0 \neq q \in Y$, we denote $\mathbb{R}_{++} q=\{r q \mid r>0\}$. The family of the neighborhood of 0 in $Y$ is denoted by $N(0)$. Let the support functional of $M$ at $m$ be defined as

$$
\sigma_{M}(\varphi)=\sup _{m \in M}\{\langle m, \varphi\rangle\}, \quad \varphi \in Y^{*}
$$

The positive dual cone of $M \subseteq Y$ is defined as

$$
M^{+}=\left\{y^{*} \in Y^{*} \mid\left\langle y^{*}, y\right\rangle \geq 0, \forall y \in M\right\},
$$

and the strict positive dual cone of $M$ is defined as

$$
M^{+i}=\left\{y^{*} \in Y^{*} \mid\left\langle y^{*}, y\right\rangle>0, \forall y \in M \backslash\{0\}\right\} .
$$


The cone generated by the set $M$ is defined as cone $M=\bigcup_{\alpha \geq 0} \alpha M$.

Let $K \subseteq Y$ and $P \subseteq Z$ be nontrivial pointed closed convex cones with a nonempty topological interior. For any given $x, y \in Y, x \leq_{K} y \Leftrightarrow y-x \in K$.

A set $C$ is called a co-radiant set if $\lambda c \in C, \forall c \in C, \forall \lambda>1$. Moreover, we assume that $C$ is a proper pointed co-radiant set and int $C \neq \emptyset$. We denote $C(\varepsilon)=\varepsilon C, \forall \varepsilon>0$, and

$$
C(0)=\bigcup_{\varepsilon>0} C(\varepsilon)
$$

Definition 2.1 (see [1]) Let $B$ be a nonempty convex subset of the cone $K . B$ is a base of $K$ if $K=\operatorname{cone} B$ and $0 \notin \operatorname{cl} B$.

Definition 2.2 (see [3]) Let $M \subseteq Y$ be a nonempty set. $\bar{y} \in M$ is called a super efficient point of $M$ if for any given $V \in N(0)$, there exists $U \in N(0)$ such that

$$
\operatorname{cl}(\operatorname{cone}(M-\bar{y})) \cap(U-K) \subseteq V
$$

and we denote it by $\bar{y} \in \operatorname{Se}(M, K)$.

Definition 2.3 (see [14]) Let $E$ be a nonempty subset in $Y$. $E$ is called an improvement set with respect to $K$ if and only if $0 \notin E$ and $E+K=E$.

The family of the improvement sets in $Y$ is denoted by $\mathfrak{T}_{Y}$.

Definition 2.4 (see [22]) Let $E \in \mathfrak{T}_{Y}$ and a nonempty set $M \subseteq Y . \bar{y} \in M$ is called an $E$ super efficient point of $M$, if for any given $V \in N(0)$, there exists $U \in N(0)$ such that

$$
\operatorname{cl}(\operatorname{cone}(M+E-\bar{y})) \cap(U-K) \subseteq V .
$$

Lemma 2.1 (see [15]) If $M \subseteq Y$ and $E \in \mathfrak{T}_{Y}$, then

(i) $E+\operatorname{int} K=\operatorname{int} E$;

(ii) $\operatorname{cl}(\operatorname{cone}(M+E))=\operatorname{cl}(\operatorname{cone}(M+\operatorname{int} E))$.

Let $B$ be a base of $K$ and write

$$
B^{s t}=\left\{y^{*} \in Y^{*} \mid \text { there exists } t>0 \text {, s.t. }\left\langle b, y^{*}\right| \geq t, \forall b \in B\right\} .
$$

Lemma 2.2 (see [24]) Let $B$ be a base of $K$ and $y^{*} \in Y^{*} \backslash\{0\} . y^{*} \in B^{\text {st }}$ if and only if there exists a neighborhood $U \in N(0)$ such that $\left\langle u-b, y^{*}\right\rangle \leq 0, \forall u \in U, \forall b \in B$.

Lemma 2.3 (see [25]) Let $B$ be a base of $K$ and $y^{*} \in B^{\text {st }}$. Then $y^{*} \in K^{+}$.

Lemma 2.4 (see [23]) Let S be any nonempty set in a real topological linear space $Y$. Then

$$
\operatorname{cl}(\operatorname{cone} S)=\operatorname{cl}(\operatorname{cone}(\operatorname{cl} S))
$$

Lemma 2.5 (see [26]) Let $Y$ be a real locally convex Hausdorff topological vector space and $M, N \subseteq Y$ be two convex sets with nonempty topological interior. If $M \cap \operatorname{int} N=\emptyset$, then there exists a hyperplane in $Y$ separating $M$ and $N$. 
Lemma 2.6 (see [27]) Let $y^{*} \in K^{+} \backslash\{0\}$, if $y \in \operatorname{int} K$, then $\left\langle y, y^{*}\right\rangle>0$.

\section{A kind of unified super efficiency}

In this section, we propose a kind of unified super efficiency via Assumption (A) proposed by Flores-Bazán and Hernández in [21] and discuss some relations with other supper efficiencies.

Assumption (A) (see [21]) Let $K \subseteq Y$ be a proper (not necessarily closed or pointed) convex cone with nonempty topological interior and $S \subsetneq Y$ be such that $0 \in \partial S$ and $S+$ $\operatorname{int} K=\operatorname{int} S$, or equivalently, $S+\operatorname{int} K \subseteq S$, or equivalently, $\operatorname{cl} S+\operatorname{int} K \subseteq S$.

Several other conditions related to Assumption (A) have been considered in economic theory and optimization.

Assumption (P) (see [21]) Let $K \subseteq Y$ be a closed convex cone. A closed set $S \subsetneq Y$ satisfies the free disposal Assumption (P) if $S+K=S$.

Assumption ( $\mathbf{P}_{S}$ ) (see [21]) Let $K \subseteq Y$ be a closed convex cone. A closed set $S \subsetneq Y$ satisfies the strong free disposal Assumption $\left(\mathrm{P}_{S}\right)$ if $S+K \backslash\{0\}=\operatorname{int} S$, or equivalently, $S+K \backslash\{0\} \subseteq \operatorname{int} S$.

Assumption (B) (see [21]) Assume that $0 \neq q \in Y$ and $S \subsetneq Y$ is a proper (not necessarily closed) set such that $0 \in \partial S$ and $\operatorname{cl}(\mathcal{C}(-S))+\mathbb{R}_{++} q \subseteq \operatorname{int}(\mathcal{C}(-S))$.

Obviously, the condition $0 \in \partial S$ can be assumed after a translation whenever $S \neq Y$. Moreover, when $0 \in \partial S$, int $S \neq \emptyset$, and int $K \neq \emptyset$, it is clear that $\left(\mathrm{P}_{S}\right) \Rightarrow(\mathrm{P}) \Rightarrow(\mathrm{A}) \Rightarrow(\mathrm{B})$. But the converses may not be true. In the following, we give three examples to illustrate them, respectively.

Example 3.1 Let $Y=\mathbb{R}^{2}, K=\left\{(x, y) \in \mathbb{R}^{2} \mid y \geq 0\right\}, S=K$. Then $S+\operatorname{int} K=\operatorname{int} S$ and $S+K=S$. Therefore, $S$ satisfies Assumption (A) and Assumption (P). $S$ cannot satisfy Assumption $\left(\mathrm{P}_{S}\right)$ since $S+K \backslash\{0\}=S \neq$ int $S$.

Example 3.2 Let $Y=\mathbb{R}^{2}, K=\left\{(x, y) \in \mathbb{R}^{2} \mid y \geq-x\right\}, S=\mathcal{C}(-K) \cup\{0\}$. Then $S+\operatorname{int} K=$ int $S=\left\{(x, y) \in \mathbb{R}^{2} \mid y>-x\right\}, S+K=K \neq S=\left\{(x, y) \in \mathbb{R}^{2} \mid y>-x\right\} \cup\{0\}$. Hence, we see that $S$ satisfies Assumption (A) and cannot satisfy Assumption (P).

Example 3.3 Let $Y=\mathbb{R}^{2}, K=\left\{(x, y) \in \mathbb{R}^{2} \mid y \geq 0\right\} \cup\{(x, y) \mid y \geq x\}$, and $S=\mathcal{C}(-K) \cup(K \cap$ $(-K))$. Then $S+\operatorname{int} K=Y \neq \operatorname{int} S$ and therefore $S$ cannot satisfy Assumption (A). It is obvious that $S$ satisfies Assumption (B).

Remark 3.1 Let $S=K$ be a pointed closed convex cone. Then $S+\operatorname{int} S=\operatorname{int} S$. It follows that $S$ satisfies Assumption (A). Let $S=E \in \mathfrak{T}_{Y}$. From Lemma 2.1(i), we can see that $S$ satisfies Assumption (A).

Remark 3.2 Let $C$ be a convex co-radiant set. Then we can easily verify that $C(\varepsilon)+$ int $C(0)=$ int $C(\varepsilon)$, which implies that $C(\varepsilon)$ satisfies Assumption (A). 
Assumption (A) holds for a wide class of (not necessarily closed) sets including these classical models. Now, we introduce a new notion of super efficiency named $S$-super efficiency via Assumption (A) in a real locally convex Hausdorff topological vector space and discuss some relations with other super efficiencies.

Definition 3.1 Assume that $S$ satisfies Assumption (A) with respect to $K$ and $M \in Y$. We say $\bar{y} \in M$ is a $S$-super efficient point of $M$ if and only if for any given $V \in N(0)$, there exists $U \in N(0)$ such that

$$
\operatorname{cl}(\operatorname{cone}(M+S-\bar{y})) \cap(U-K) \subseteq V
$$

The set of $S$-super efficient points of the set $M$ is denoted by $O_{S e}^{S}(M, K)$.

Remark 3.3 It follows from Definition 3.1 that $\bar{y} \in O_{S e}^{S}(M, K)$ if and only if, for any given $V \in N(0)$, there exists $U \in N(0)$ such that

$$
\operatorname{cone}(M+S-\bar{y}) \cap(U-K) \subseteq V
$$

Remark 3.4 Let $S=E$ be an improvement set. From Lemma 2.1(i), $S$ satisfies Assumption (A). Then from Lemma 2.1(ii), the $S$-super efficiency reduces to $E$-super efficiency proposed by Zhou and Yang in [22].

Remark 3.5 Let $S=\varepsilon+K$ where $K$ is a pointed closed convex cone. Then we can easily see that $S$ satisfies Assumption (A) and hence $S$-super efficiency reduces to $\varepsilon$-super efficiency as proposed by Ling in [10].

Remark 3.6 Let $K \subseteq Y$ be a point closed convex cone with nonempty topological interior and $S=K$. Then $S$-super efficiency reduces to the classical super efficiency. In fact, since $K$ is a convex cone, we have $K+\operatorname{int} K=\operatorname{int} K$, and $0 \in \partial K$. Then it satisfies Assumption (A). Assume that $\bar{y}$ is a $S$-super efficiency point of set-valued map, and from Lemma 2.4 we have

$$
\begin{aligned}
& \operatorname{cl} \operatorname{cone}(M+S-\bar{y}) \cap(U-K) \\
& \quad=\operatorname{clcone}(M+K-\bar{y}) \cap(U-K) \subseteq V \quad \Rightarrow \quad \operatorname{clcone}(M-\bar{y}) \cap(U-K) \subseteq V,
\end{aligned}
$$

which implies that $\bar{y}$ is a super efficient point.

Theorem 3.1 Let S satisfy Assumption (A) with respect to $K, M \subseteq Y, B$ be a base of $K$, and $\bar{y} \in O_{S e}^{S}(M, K)$. Then there exists $U \in N(0)$ such that

$$
\operatorname{cl}(\operatorname{cone}(M+S-\bar{y})) \cap(U-B)=\emptyset .
$$

Proof Since $B$ is a base of $K, 0 \notin \operatorname{cl} B$. Therefore, there exists a convex balanced neighborhood $V \in N(0)$ such that $0 \notin B+V$. Write $V_{1}=\frac{1}{2} V$. Clearly,

$$
(-B) \cap\left(V_{1}+V_{1}\right)=\varnothing .
$$


From (3.1) and the fact that $V_{1}$ is balanced, it is easy to prove that

$$
\left(V_{1}-B\right) \cap V_{1}=\emptyset .
$$

By $\bar{y} \in O_{S e}^{S}(M, K)$ and $V_{1} \in N(0)$, there exists a convex neighborhood $U_{1} \in N(0)$ such that

$$
\operatorname{cl}(\operatorname{cone}(M+S-\bar{y})) \cap\left(U_{1}-K\right) \subseteq V_{1} .
$$

Write $U=U_{1} \cap V_{1}$. Clearly, $U$ is a convex neighborhood in $N(0)$. Since $U \subseteq U_{1}$, it follows that

$$
\operatorname{cl}(\operatorname{cone}(M+S-\bar{y})) \cap(U-K) \subseteq V_{1} .
$$

Since $U \subseteq V_{1}$, it follows from (3.2) that

$$
(U-B) \cap V_{1}=\emptyset .
$$

Clearly,

$$
\operatorname{cl}(\operatorname{cone}(M+S-\bar{y})) \cap(U-B) \subseteq U-B .
$$

By (3.3), we obtain

$$
\operatorname{cl}(\operatorname{cone}(M+S-\bar{y})) \cap(U-B) \subseteq \operatorname{cl}(\operatorname{cone}(M+S-\bar{y})) \cap(U-K) \subseteq V_{1} .
$$

Using (3.4), (3.5), and (3.6), we have $\operatorname{cl}(\operatorname{cone}(M+S-\bar{y})) \cap(U-B)=\emptyset$.

\section{Scalarization theorems}

Theory and practice of vector optimization have always been closely related to scalarization procedures. The one most widely used is probably the linear scalarization, by which the properly efficient points can be characterized (under convexity assumptions) as the solutions of a linearly scalarized problem.

In this section, we establish scalarization theorems of $S$-super efficiency for a vector optimization problem with set-valued maps. Let $F: I \rightrightarrows Y$ and $G: I \rightrightarrows Z$ be two set-valued maps. Consider the following vector optimization problems with set-valued maps:

$$
\begin{aligned}
& \text { (VP) } \min F(x) \\
& \text { s.t. } x \in D=\{x \in I \mid G(x) \cap(-P) \neq \emptyset\} .
\end{aligned}
$$

The corresponding scalar optimization problem of (VP) is defined as follows:

$$
\begin{array}{cl}
(\mathrm{VP})_{\varphi} \quad & \min \langle F(x), \varphi\rangle \\
& \text { s.t. } x \in D,
\end{array}
$$

where $\varphi \in Y^{*} \backslash\{0\}$. 
Definition 4.1 Let $S \subsetneq Y$ satisfy Assumption (A) with respect to $K, \bar{x} \in D$ is a $S$-super efficient solution of (VP) if and only if there exists $\bar{y} \in F(\bar{x})$ such that $\bar{y} \in O_{S e}^{S}(F(D))$. The point pair $\langle\bar{x}, \bar{y}\rangle$ is called a $S$-super efficient element of (VP).

Definition 4.2 A point $\bar{x} \in D$ is called an optimal solution of $(\mathrm{VP})_{\varphi}$ with respect to $S$ which satisfies Assumption (A) with respect to $K$, if there exists $\bar{y} \in F(\bar{x})$ such that

$$
\langle y-\bar{y}, \varphi\rangle \geq \sigma_{-S}(\varphi), \quad \forall x \in D, \forall y \in F(x)
$$

The point pair $(\bar{x}, \bar{y})$ is called a $S$-optimal point of $(\mathrm{VP})_{\varphi}$.

Definition 4.3 Let $D \subseteq Y$ and $S \subsetneq Y$ satisfy Assumption (A) with respect to $K$. The setvalued map $F: D \rightrightarrows Y$ is called nearly $S$-subconvexlike on $D$ if and only if cl $(\operatorname{cone}(F(D)+S))$ is a convex set in $Y$.

Remark 4.1 If $S=E \in \mathfrak{T}_{Y}$, then nearly $S$-subconvexlikeness coincides with nearly $E$ subconvexlikeness as proposed by Zhao et al. in [15].

Remark 4.2 If $C$ is a convex co-radiant set, $\varepsilon \geq 0$, and $S=C(\varepsilon)$, then nearly $S$ subconvexlikeness coincides with nearly $C$-subconvexlikeness as in [20].

Remark 4.3 If $S=K$ is a pointed closed convex cone, then nearly $S$-subconvexlikeness coincides with nearly $K$-subconvexlikeness as in [23].

Theorem 4.1 Let $B$ be a base of $K$ and $S$ satisfy the Assumption (A) with respect to $K$. Suppose that the following conditions hold:

(i) $(\bar{x}, \bar{y})$ is a S-super efficient element of (VP);

(ii) $F-\bar{y}$ is nearly S-subconvexlike on $D$.

Then there exists $\varphi \in B^{\text {st }}$ such that $(\bar{x}, \bar{y})$ is a S-optimal element of $(\mathrm{VP})_{\varphi}$.

Proof By condition (i) and the proof of Theorem 3.1, there exists a convex neighborhood in $N(0)$, such that

$$
\operatorname{cl}(\operatorname{cone}(F(D)+S-\bar{y})) \cap(U-B)=\emptyset .
$$

Since $F-\bar{y}$ is nearly $S$-subconvexlike on $D, \operatorname{cl}(\operatorname{cone}(F(D)+S-\bar{y}))$ is a convex set in $Y$. Clearly, $U-B$ is a nonempty open convex set in $Y$. By Lemma 2.5, there exists $\varphi \in Y^{*} \backslash\{0\}$ such that

$$
\left\langle y_{1}, \varphi\right\rangle \geq\left\langle y_{2}, \varphi\right\rangle, \quad \forall y_{1} \in \operatorname{cl}(\operatorname{cone}(F(D)+S-\bar{y})), \forall y_{2} \in U-B
$$

Since $0 \in \operatorname{cl}(\operatorname{cone}(F(D)+S-\bar{y}))$, it follows that

$$
\left\langle y_{2}, \varphi\right\rangle \leq 0, \quad \forall y_{2} \in U-B
$$

From Lemma 2.2, $\varphi \in B^{s t}$. 
By (4.1), $\varphi$ is bounded below on $\operatorname{cl}(\operatorname{cone}(F(D)+S-\bar{y}))$. Since $\operatorname{cl}(\operatorname{cone}(F(D)+S-\bar{y}))$ is a cone in $Y$, it follows from (4.1) that

$$
\left\langle y_{1}, \varphi\right\rangle \geq 0, \quad \forall y_{1} \in \operatorname{cl}(\operatorname{cone}(F(D)+S-\bar{y}))
$$

Clearly

$$
\left\langle y_{1}, \varphi\right\rangle \geq 0, \quad \forall y_{1} \in F(D)+S-\bar{y}
$$

Hence

$$
\langle y, \varphi\rangle+\langle s, \varphi\rangle \geq\langle\bar{y}, \varphi\rangle, \quad \forall x \in D, \forall y \in F(x), \forall s \in S .
$$

Then we have

$$
\langle y, \varphi\rangle-\langle\bar{y}, \varphi\rangle \geq \sigma_{-S}(\varphi), \quad \forall x \in D, \forall y \in F(x),
$$

which implies that $(\bar{x}, \bar{y})$ is a $S$-optimal element of $(\mathrm{VP})_{\varphi}$.

Remark 4.4 Theorem 4.1 is a generalization of Corollary 2.3 in [1], Theorem 3.1 in [10], Theorem 4.1 in [22], and Theorem 2.1 in [28].

Theorem 4.2 Let $B$ be a bounded base of $K$ and $S$ satisfy Assumption (A) with respect to $K$. If there exists $\varphi \in B^{\text {st }}$ such that $(\bar{x}, \bar{y})$ is a S-optimal element of $(\mathrm{VP})_{\varphi}$, then $(\bar{x}, \bar{y})$ is a $S$-super efficient element of (VP).

Proof Suppose that $(\bar{x}, \bar{y})$ is not a $S$-super efficient element of (VP). Therefore, $\bar{y} \notin$ $O_{S e}^{S}(F(D))$. By Remark 3.3 there exists $V_{1} \in N(0)$, such that

$$
\text { cone }(F(D)+S-\bar{y}) \cap(U-K) \nsubseteq V_{1}, \quad \forall U \in N(0)
$$

Thus, for any $U \in N(0)$, there exists $a_{U} \in U, \lambda_{U} \geq 0, b_{U} \in B$, such that

$$
a_{U}-\lambda_{U} b_{U} \in \operatorname{cone}(F(D)+S-\bar{y})
$$

and

$$
a_{U}-\lambda_{U} b_{U} \notin V_{1} \text {. }
$$

For $V_{1}$, there exists $V_{2} \in N(0)$ such that

$$
V_{2}-V_{2} \subseteq V_{1}
$$

without loss of generality, we may assume that $\left\{a_{U} \mid U \in N(0)\right\} \subseteq V_{2}$. Clearly, $\lim _{U} a_{U}=0$. For any $U \in N(0)$, it follows from (4.3) and (4.4) that $\lambda_{U} b_{U} \notin V_{2}$. Since $B$ is bounded, there exists $\lambda_{0}>0$ such that

$$
\lambda B \subseteq V_{2}, \quad \lambda \in\left[0, \lambda_{0}\right]
$$


It is easy to check that $\lambda_{U}>\lambda_{0}$ for any $U \in N(0)$. It follows from (4.2) that there exist $r_{U}>0, y_{U} \in F(D)$ and $s_{U} \in S$, such that

$$
a_{U}-\lambda_{U} b_{U}=r_{U}\left(y_{U}+s_{U}-\bar{y}\right) .
$$

Since $(\bar{x}, \bar{y})$ is an $S$-optimal element of $(\mathrm{VP})_{\varphi}$, it follows from (4.5) that

$$
\left\langle a_{U}-\lambda_{U} b_{U}, \varphi\right\rangle=\left\langle r_{U}\left(y_{U}+s_{U}-\bar{y}\right), \varphi\right\rangle=r_{U}\left\langle y_{U}+s_{U}-\bar{y}, \varphi\right\rangle \geq 0, \quad \forall U \in N(0),
$$

i.e.,

$$
\left\langle a_{U}, \varphi\right\rangle \geq \lambda_{U}\left\langle b_{U}, \varphi\right\rangle, \quad \forall U \in N(0) .
$$

Because $\varphi \in B^{s t}$, there exists $t>0$, such that

$$
\langle b, \varphi\rangle \geq t, \quad \forall b \in B .
$$

Then we obtain

$$
\left\langle a_{U}, \varphi\right\rangle \geq \lambda_{U} t>\lambda_{0} t>0, \quad \forall U \in N(0)
$$

Limiting (4.6), we have $0=\lim _{U}\left\langle a_{U}, \varphi\right\rangle \geq \lambda_{0} t>0$. This is a contradiction. Therefore, $(\bar{x}, \bar{y})$ is a $S$-super efficient element of (VP).

\section{Lagrange multipliers theorems}

In this section, we establish Lagrange multipliers theorems of $S$-super efficiency under the nearly $S$-subconvexlikeness. Let $L(Z, Y)$ be the set of all linear continuous operators from $Z$ to $Y$. A subset $L^{+}(Z, Y)$ of $L(Z, Y)$ is defined as

$$
L^{+}(Z, Y)=\{T \in L(Z, Y) \mid T(D) \subseteq K\} .
$$

The Lagrange set-valued map of (VP) is defined by

$$
L(x, T)=F(x)+T(G(x)), \quad \forall(x, T) \in D \times L^{+}(Z, Y) .
$$

Consider the following vector optimization problem:

$$
\begin{array}{ll}
(\mathrm{UVP})_{T} & \min L(x, T) \\
& \text { s.t. }(x, T) \in D \times L^{+}(Z, Y) .
\end{array}
$$

Assume that $I(x)=F(x) \times G(x)$ is the set-valued map with $I: D \rightrightarrows Y \times Z . I(x)$ is nearly $S \times P$-subconvexlike on $D$ if and only if $\operatorname{cl}(\operatorname{cone}(I(D)+S \times P))$ is a convex set in $Y \times Z$.

Theorem 5.1 Let B be a bounded base of $K$ and S satisfy Assumption (A) with respect to $K$. Let $\bar{x} \in D$ and $0 \in G(\bar{x})$. Suppose the following conditions hold:

(i) $(\bar{x}, \bar{y})$ is a S-super efficient element of (VP); 
(ii) $\bar{I}(x)$ is nearly $S \times P$-subconvexlike on $D$, where

$$
\bar{I}(x)=(F(x)-\bar{y}) \times G(x)
$$

(iii) $\operatorname{cl}(\operatorname{cone}(G(D)+P))=Z$.

Then there exists $\bar{T} \in L^{+}(Z, Y)$ such that

(a) $-\bar{T}(G(\bar{x}) \cap(-P)) \subseteq(\operatorname{int} K \cup\{0\}) \backslash \operatorname{int} S$;

(b) $(\bar{x}, \bar{y})$ is a S-super efficient element of $(\mathrm{UVP})_{\bar{T}}$.

Proof (a) Since $(\bar{x}, \bar{y})$ is a $S$-super efficient element of (VP), from Theorem 3.1, there exists a convex neighborhood $U \in N(0)$, such that

$$
\operatorname{cl}(\operatorname{cone}(F(D)+S-\bar{y})) \cap(U-B)=\emptyset .
$$

Then

$$
\operatorname{cone}(F(D)+S-\bar{y}) \cap(U-B)=\emptyset .
$$

Then it is easy to prove that

$$
\operatorname{cone}(\bar{I}(D)+S \times P) \cap((U-B) \times(-\operatorname{int} P))=\emptyset .
$$

Since $(U-B) \times(-\operatorname{int} P)$ is an open convex set in $Y \times Z$,

$$
\operatorname{cl}(\operatorname{cone}(\bar{I}(D)+S \times P)) \cap((U-B) \times(-\operatorname{int} P))=\emptyset .
$$

By condition (ii), $\operatorname{cl}(\operatorname{cone}(\bar{I}(D)+S \times P))$ is a nonempty convex set in $Y \times Z$. Then from Lemma 2.5 there exists $\left(y^{*}, z^{*}\right) \in\left(Y^{*} \times Z^{*}\right) \backslash\left\{\left(0_{Y}^{*}, 0_{Z}^{*}\right)\right\}$, for any $\lambda \geq 0, x \in D,(y, z) \in F(x) \times$ $G(x), s \in S, p \in P$, and $\left(u, p^{\prime}\right) \in(U-B) \times(-\operatorname{int} P)$ such that

$$
\lambda\left\langle y-\bar{y}+s, y^{*}\right\rangle+\lambda\left|z+p, z^{*}\right\rangle \geq\left\langle u, y^{*}\right\rangle+\left\langle p^{\prime}, z^{*}\right\rangle
$$

Then

$$
\lambda\left\langle y-\bar{y}+s, y^{*}\right\rangle+\lambda\left|z, z^{*}\right\rangle+\left\langle u^{\prime}, y^{*}\right\rangle+\left\langle p^{\prime \prime}, z^{*}\right\rangle \geq 0,
$$

where $p^{\prime \prime}=\lambda p-p^{\prime} \in P+\operatorname{int} P=\operatorname{int} P, u^{\prime}=-u \in B-U$.

Let $\lambda=0$ in (5.1), we have

$$
\left\langle u^{\prime}, y^{*}\right\rangle+\left\langle p^{\prime \prime}, z^{*}\right\rangle \geq 0, \quad \forall\left(u^{\prime}, p^{\prime \prime}\right) \in(B-U) \times \operatorname{int} P .
$$

Because $P$ is a cone in $Z$,

$$
\left\langle u^{\prime}, y^{*}\right\rangle \geq 0, \quad \forall u^{\prime} \in B-U, \quad \text { and } \quad\left\langle p^{\prime \prime}, z^{*}\right\rangle \geq 0, \quad \forall p^{\prime \prime} \in \operatorname{int} P .
$$

So, from Lemma 2.2, $y^{*} \in B^{s t}, z^{*} \in P^{+}$. Fixing $u^{\prime}$ and $p^{\prime \prime}$ in (5.1), we have

$$
\left\langle y-\bar{y}+s, y^{*}\right\rangle+\left\langle z, z^{*}\right\rangle+\frac{1}{\lambda}\left(\left\langle u^{\prime}, y^{*}\right\rangle+\left\langle p^{\prime \prime}, z^{*}\right\rangle\right) \geq 0 .
$$


Let $\lambda \rightarrow+\infty$ in (5.2), it follows that

$$
\left\langle y-\bar{y}+s, y^{*}\right\rangle+\left\langle z, z^{*}\right\rangle \geq 0, \quad \forall x \in D, \forall(y, z) \in F(x) \times G(x), \forall s \in S
$$

we can prove that $y^{*} \neq 0$. Otherwise, $z^{*} \neq 0$, then

$$
\left\langle z, z^{*}\right\rangle \geq 0, \quad \forall x \in D, z \in G(x)
$$

From $z^{*} \in P^{+}$, we have $z^{*} \in \operatorname{cl}(\operatorname{cone}(G(D)+P))^{+}$. By condition (iii), $z^{*} \in Z^{+}$. Then $z^{*}=0$, which is a contradiction. Hence, $y^{*} \neq 0$. From $y^{*} \in B^{s t}$, there exists $k_{1} \in \operatorname{int} K$, which satisfies $\left\langle k_{1}, y^{*}\right\rangle=1$.

Define the map $\bar{T}: Z \rightrightarrows Y$ as follows:

$$
\bar{T}(z)=\left\langle z, z^{*}\right| k_{1}, \quad \forall z \in Z
$$

Then $\bar{T} \in L^{+}(Z, Y)$. Since $0 \in G(x), \bar{y} \in F(\bar{x}) \subseteq F(\bar{x})+\bar{T}(G(\bar{x}))$. Setting $x=\bar{x}$ and $y=\bar{y}$ in (5.3), we have

$$
\left\langle-s, y^{*}\right\rangle \leq\left\langle\bar{z}, z^{*}\right\rangle \leq 0, \quad \forall s \in S, \bar{z} \in G(\bar{x}) \cap(-P) .
$$

Then

$$
-\bar{T}(\bar{z})=-\left(\bar{z}, z^{*}\right) k_{1} \in \operatorname{int} K \cup\{0\}, \quad \forall \bar{z} \in G(\bar{x}) \cap(-P) .
$$

In the following, we prove that

$$
-\bar{T}(\bar{z}) \notin \operatorname{int} S, \quad \forall \bar{z} \in G(\bar{x}) \cap(-P) .
$$

Otherwise, there exists $\tilde{z} \in G(\bar{x}) \cap(-P)$ such that $-\bar{T}(\tilde{z}) \in \operatorname{int} S$. From $S$ satisfying Assumption (A), there exists $s \in S, k \in \operatorname{int} K$ such that

$$
-\bar{T}(\tilde{z})-s=k \in \operatorname{int} K \text {. }
$$

From Lemmas 2.3 and 2.6, we have $y^{*} \in K^{+} \backslash\{0\}$, then $\left\langle-\bar{T}(\tilde{z})-s, y^{*}\right\rangle>0$, i.e., $\left\langle-s, y^{*}\right\rangle>$ $\left\langle\tilde{z}, z^{*}\right\rangle$, which contradicts (5.5). Then (5.6) is true. Therefore, $-\bar{T}(G(-\bar{x}) \cap(-P)) \subseteq \operatorname{int} K \cup$ $\{0\} \backslash \operatorname{int} S$.

(b) Since $0 \in G(\bar{x})$, from (5.3) and (5.4) we have

$$
\begin{aligned}
\left\langle\bar{y}+\bar{T}(0), y^{*}\right\rangle & =\left\langle\bar{y}, y^{*}\right\rangle \leq\left\langle y+s, y^{*}\right\rangle+\left\langle z, z^{*}\right\rangle \\
& =\left\langle y+s, y^{*}\right\rangle+\left\langle z, z^{*}\right\rangle\left\langle k_{1}, y^{*}\right\rangle \\
& =\left\langle y+s, y^{*}\right\rangle+\left\langle\left\langle z, z^{*}\right\rangle k_{1}, y^{*}\right\rangle \\
& =\left\langle y+\bar{T}(z)+s, y^{*}\right\rangle \quad(\forall x \in D, \forall y \in F(x), \forall z \in G(x), \forall s \in S) .
\end{aligned}
$$

Then we have

$$
\left\langle(y+\bar{T}(z))-(\bar{y}+\bar{T}(0)), y^{*}\right\rangle \geq\left\langle-s, y^{*}\right\rangle \quad(\forall x \in D, \forall y \in F(x), \forall z \in G(x), \forall s \in S),
$$


i.e.,

$$
\left\langle(y+\bar{T}(z))-(\bar{y}+\bar{T}(0)), y^{*}\right\rangle \geq \sigma_{-S}\left(y^{*}\right) \quad(\forall x \in D, y \in F(x), z \in G(x)) .
$$

It follows that $(\bar{x}, \bar{y})$ is a $S$-optimal element of the problem

$$
\min \left\langle L\left(x, \bar{T}, y^{*}\right)\right\rangle \text { subject to } x \in D \text {. }
$$

According to Theorem 4.2, $(\bar{x}, \bar{y})$ is a $S$-super efficient element of (UVP) $)_{\bar{T}}$.

Remark 5.1 Theorem 5.1 is a generalization of Theorem 6 in [2], Theorem 5.1 in [4], Theorem 4.1 in [5], Theorem 4.1 in [10], Theorem 4.1 in [22], and Theorem 4.1 in [29].

Theorem 5.2 Let $S \subsetneq Y$ satisfy Assumption (A) with respect to $K$ and $\bar{x} \in D, \bar{y} \in F(\bar{x})$. If there exists $\bar{T} \in L^{+}(Z, Y)$ such that $(\bar{x}, \bar{y})$ is a S-super efficient element of $(\mathrm{UVP})_{\bar{T}}$, then $(\bar{x}, \bar{y})$ is a $S$-super efficient element of $(\mathrm{VP})$.

Proof Since $(\bar{x}, \bar{y})$ is a $S$-super efficient element of $(\mathrm{UVP})_{\bar{T}}$, for any neighborhood $V \in N(0)$, there exists a neighborhood $U \in N(0)$ such that

$$
\operatorname{cl}\left(\operatorname{cone}\left(\bigcup_{x \in D}(F(x)+\bar{T}(G(x))+S-\bar{y})\right)\right) \cap(U-K) \subseteq V .
$$

Let $x \in D$. Taking $z \in G(x) \cap(-P)$, we have $-\bar{T}(z) \in \operatorname{int} K \cup\{0\}$. Then $0 \in \bar{T}(G(x))+($ int $K) \cup$ $\{0\}$. Hence, from $S$ satisfying Assumption (A), we have

$$
\begin{aligned}
F(x)+S-\bar{y} & \subseteq F(x)+S+\bar{T}(G(x))+(\operatorname{int} K \cup\{0\})-\bar{y} \\
& =F(x)+\bar{T}(G(x))+S-\bar{y} .
\end{aligned}
$$

It follows that

$$
\operatorname{cl}(\operatorname{cone}(F(D)+S-\bar{y})) \subseteq \operatorname{cl}\left(\operatorname{cone}\left(\bigcup_{x \in D}(F(x)+\bar{T}(G(x))+S-\bar{y})\right)\right) .
$$

From (5.7), we have

$$
\operatorname{cl}(\operatorname{cone}(F(D)+S-\bar{y})) \cap(U-K) \subseteq V .
$$

Then $(\bar{x}, \bar{y})$ is a $S$-super efficient element of (VP).

Remark 5.2 When $S=K \backslash\{0\}$, Theorem 5.2 reduces to Theorem 4.2 in [7]. Furthermore, the condition $\bar{T}(G(\bar{x}) \cap(-P))=\{0\}$ is removed.

In this paper, we established scalarization theorems and Lagrange multiplier theorems of $S$-super efficiency under the assumption of nearly $S$-subconvexlikeness. It will be a future study to use the main results to derive the $S$-super saddle point theorem, $S$-super duality results including weak duality, and strong duality theorems of the vector optimization with set-valued maps. We believe that the results have both potential consequences for theoretical and numerical investigations in vector optimization problems. 


\section{Competing interests}

The authors declare that they have no competing interests.

\section{Authors' contributions}

All authors contributed equally and significantly in writing this article. All authors read and approved the final manuscript.

\section{Acknowledgements}

This work is partially supported by the National Natural Science Foundation of China (Grants: 11301574, 11271391,

11431004) and the Second Sponsoring Plan for Young Key Teachers from Universities of Chongqing.

\section{Received: 11 December 2014 Accepted: 11 May 2015 Published online: 28 May 2015}

\section{References}

1. Borwein, JM, Zhuang, DM: Supper efficiency in vector optimization. Trans. Am. Math. Soc. 338(1), 105-122 (1993)

2. Borwein, JM, Zhuang, DM: Supper efficiency in convex vector optimization. ZOR, Z. Oper.-Res. 35, 175-184 (1991)

3. Zheng, XY: Proper efficiency in locally convex topological vector spaces. J. Optim. Theory Appl. 94(2), 469-486 (1997)

4. Rong, WD, Wu, YN: Characterizations of super efficiency in cone-convexlike vector optimization with set-valued maps. Math. Methods Oper. Res. 48(2), 247-258 (1998)

5. Mehra, A: Super efficiency in vector optimization with nearly convexlike set-valued maps. J. Math. Anal. Appl. 276(2), 815-832 (2002)

6. Qiu, QS, Fu, WT: The connectedness of the super efficient solution sets of the optimization problem for a set-valued mapping. J. Syst. Sci. Math. Sci. 22(1), 107-114 (2002)

7. Xia, LY, Qiu, JH: Superefficiency in vector optimization with nearly subconvexlike set-valued maps. J. Optim. Theory Appl. 136(1), 125-137 (2008)

8. Kutateladze, SS: Convex $\varepsilon$-programming. Sov. Math. Dokl. 20(2), 391-393 (1979)

9. Rong, WD, Wu, YN: $\varepsilon$-Weak minimal solutions of vector optimization problems with set-valued maps. J. Optim. Theory Appl. 48(2), 247-258 (1998)

10. Ling, C: $\varepsilon$-Super efficient solutions of vector optimization problems with set-valued maps. Oper. Res. Trans. 5(3), $51-56(2001)$

11. Ling, C: $\varepsilon$-Super saddle points and $\varepsilon$-duality theorems of vector optimization problems with set-valued maps. Oper Res. Trans. 6(1), 53-60 (2002)

12. Tuan, LA: $\varepsilon$-Optimality conditions for vector optimization problems with set-valued maps. Numer. Funct. Anal. Optim 31(1), 78-95 (2010)

13. Chicco, M, Mignanego, F, Pusillo, L, Tijs, S: Vector optimization problems via improvement sets. J. Optim. Theory Appl. 150(3), 516-529 (2011)

14. Gutiérrez, C, Jiménez, B, Novo, V: Improvement sets and vector optimization. Eur. J. Oper. Res. 223(2), 304-311 (2012)

15. Zhao, KQ, Yang, XM, Peng, JW: Weak E-optimal solution in vector optimization. Taiwan. J. Math. 17(4), 1287-1302 (2013)

16. Zhao, KQ, Yang, XM: E-Benson proper efficiency in vector optimization. Optimization (2013) doi:10.1080/02331934.2013.798321

17. Zhao, KQ, Yang, XM: Characterizations of the E-Benson proper efficiency in vector optimization problems. Numer Algebra Control Optim. 3(4), 643-653 (2013)

18. Gutiérrez, C, Jiménez, B, Novo, V: A unified approach and optimality conditions for approximate solutions of vector optimization problems. SIAM J. Optim. 17(3), 688-710 (2006)

19. Gutiérrez, C, Jiménez, B, Novo, V: Optimality conditions via scalarization for a new $\varepsilon$-efficiency concept in vector optimization problems. Eur. J. Oper. Res. 201(1), 11-22 (2010)

20. Gao, Y, Yang, XM, Yang, Y, Yan, H: Scalarization and Lagrange multipliers for approximate solutions in the vector optimization problems with set-valued maps. J. Ind. Manag. Optim. 11(2), 673-683 (2015)

21. Flores-Bazán, F, Hernández, E: A unified vector optimization problem: complete scalarizations and applications. Optimization 60(12), 1399-1419 (2011)

22. Zhou, ZZ, Yang, XM: E-Super efficiency of set-valued optimization problems involving improvement sets. J. Ind. Manag. Optim. (accepted)

23. Yang, XM, Li, D, Wang, SY: Near-subconvexlikeness in vector optimization with set-valued functions. J. Optim. Theory Appl. 110(2), 413-427 (2001)

24. Cheng, YH, Fu, WT: Strong efficiency in a locally convex space. Math. Methods Oper. Res. 50(3), 373-384 (1999)

25. Fu, WT, Cheng, YH: On the strict efficiency in a locally convex space. J. Syst. Sci. Complex. 12(1), 40-44 (1999)

26. Tiel, JV: Convex Analysis. Wiley, New York (1984)

27. Li, ZM: A theorem of the alternative and its application to the optimization of set-valued maps. J. Optim. Theory Appl. 100(2), 365-375 (1999)

28. $\mathrm{Hu}, \mathrm{YD}, \mathrm{Gong}, \mathrm{XH}$ : Super efficiency and its scalarization in topological vector space. Acta Math. Appl. Sin. 16(1), 22-26 (2000)

29. $\mathrm{Xu}, \mathrm{YH}, \mathrm{Liu}, \mathrm{SY}$ : Super efficiency in the nearly cone-subconvexlike vector optimization with set-valued functions. Acta Math. Sci. 25(1), 152-160 (2005) 